\title{
CIENCIA Y VALORES EN LOS ESTUDIOS DEL CEREBRO ${ }^{1}$
}

\section{Amparo Gómez Rodríguez}

\begin{abstract}
In this paper the biological theories of the XIX century about the mental inferiority of the women are examined. The analysis is centered in evolutionism, phrenology and studies of the brain (with special attention to the proposal of Moebius). The author analyzes these theories showing that the ideology about the inferiority of women acts in the hypotheses that are sustained and in researchs that are carried out. The ideology acts in background assumptions and external values on women that are operating in scientific practice in these scientific areas. The conclusion is that these theories confirm the dominant gender stereotypes.

KEY WORDS Contextual and constitutive values. Phrenology. Gender.
\end{abstract}

RESUMEN En este artículo se examinan las teorias biológicas del siglo XIX acerca de la inferioridad mental de las mujeres. El estudio se centra en el evolucionismo, la frenología y los estudios del cerebro (con especial atención a la propuesta de Moebius). Se lleva a cabo un análisis de dichas teorias mostrando como opera la ideología acerca de la inferioridad de las mujeres tanto en las hipótesis que se sostienen como en las investigaciones que se llevan a cabo. La ideología se concreta en el trasfondo de asunciones y valores externas que está operando en la práctica científica llevada a cabo en estas áreas científicas. La conclusión es que tales teorias se limitan a confirmar los estereotipos de género dominantes.

PALABRAS CLAVE Valores contextuales y constitutivos. Frenología. Género.

\section{Introducción}

La filosofia, la historia y los estudios sociales de la ciencia de las últimas décadas han mostrado que el conocimiento científico no es axiológicamente neutro. La ciencia incluye valores epistémicos plurales y está sometida, en distintos grados, a la influencia de factores externos de diversa naturaleza. En este contexto los estudios feministas de la ciencia y la tecnología han destacado la existencia de valores de género que inciden sobre la ciencia sesgándola profundamente $^{2}$. Los trabajos llevados a cabo en este terreno coinciden en señalar que de una forma u otra la ciencia ha resultado permeada por los valores androcéntricos dominantes en la cultura occidental en cuyo seno se ha constituido ${ }^{3}$. Esto ha dado lugar a una revisión crítica del conocimiento científico desde posiciones epistemológicas diversificadas $y$, en muchos casos, a un cuestionamiento en profundidad del modelo de ciencia existente ${ }^{4}$.

Uno de los planteamientos más interesantes en este contexto es el que sostiene Helen Longino y que ella ha denominado empirismo contextual ${ }^{5}$. Este enfoque se distancia, por un lado, de las posiciones externalistas que reducen la ciencia a mera ideologia mientras que, por otro, se opone a las tesis empiristas clásicas que ven en el recurso al método científico la posibilidad de una ciencia neutra y objetiva, libre de incidencias externas.

Según Longino, la ciencia no se limita a ser un producto social resultado de los valores culturales dominantes y de la subjetividad que afecta a sus perspectivas y método, pero tampoco es cierto que el método científico evite la incidencia de externalidades, pues "los componentes básicos de las metodologías -lógica y observación- no son suficientes para excluir los valores de la investigación" ${ }^{6}$. Estos no son eliminables a través de una práctica científica supuestamente correcta que garantice la buena ciencia, pues la cuestión no es de buena o mala ciencia, ya que la mejor ciencia no está libre de la influencia de factores externos ${ }^{7}$. Pensar lo contrario es sostener una visión enormemente simplista del conocimiento científico como conjunto de teorias justificadas por recurso al método que aparece así como garante del incremento histórico de la objetividad. La ciencia poco tiene que ver con esta imagen; según Longino se caracteriza por ser una actividad desarrollada por una comunidad científica (una de cuyas caracteristicas centrales es su capaci- 
dad de ejercer la crítica) en contextos específicos y porque tal práctica es llevada a cabo desde unos valores y supuestos de base 0 de trasfondo (background assumptions) ${ }^{8}$.

La noción de "supuestos de trasfondo" es fundamental en el pensamiento de Longino. Refiere a valores epistémicos, normas acerca de qué constituye una práctica cientifica aceptable, reglas de procedimiento, supuestos ontológicos o acerca de los fines de la ciencia, presupuestos teóricos 0 metateóricos, y asunciones sobre las hipótesis, todo ello compartido por la comunidad científica. Pero también refiere a la existencia, al menos a veces, de valores externos 0 contextuales en la terminologia de la autora ${ }^{9}$.

Longino mantiene que incluso el criterio de adecuación empírica, uno de los valores epistémicos que ella sostiene, está mediatizado por este trasfondo. Esto es asi porque un "estado de cosas" es evidencia empírica relevante para una hipótesis o teoría, sólo a la luz de los supuestos de trasfondo que afirman una conexión entre el tipo de datos logrados y los procesos o estados descritos por las hipótesis ${ }^{10}$. La evidencia empírica no es relevante por si misma sino desde este trasfondo en contextos de investigación específicos. Los mismos datos pueden ser evidencia para hipótesis distintas, e incluso opuestas. No hay una única relación entre estados de cosas e hipótesis y no hay algo así como una relación natural, causal, entre ambas. Son las creencias de las personas concernidas con la relación evidencial las que cuentan. Por tanto, las teorias tienen un alto grado de indeterminación por los hechos ${ }^{11}$.

En la medida en que los supuestos de trasfondo incluyen valores internos pero también externos (constitutivos y contextuales en la terminología de la autora ${ }^{12}$ ), Longino afirma que la conexión entre datos e hipótesis puede ser permeable a valores externos y la mejor ciencia no puede evitar este hecho. Los cientificos pueden estar aplicando las reglas más estrictas del método $y_{1}$ sin embargo, hacerlo desde un background que incluye asunciones externas. Las mediciones (en el s. XIX) de indices cefálicos, peso y volumen del cerebro, o las observaciones de Rüdinger sobre las circunvoluciones cerebrales, se llevaron a cabo aplicando estrictamente el método científico tal como se le concebia en ese momento (eran buena ciencia) pero esto se hizo desde asunciones racistas y sexistas que determinaron las teorias en cuestión.

Esto no significa que la influencia de lo externo afecte a todas las ciencias por igual, ni en el mismo grado. En cier-

ARBOR CLXXXI 716 NOVIEMBRE-DICIEMBRE (2005) 479-492 ISSN: 0210-1963 tos ámbitos del conocimiento científico los supuestos de trasfondo pueden no incluir valores externos ${ }^{13}$. Longino explica este hecho señalando que hay realidades que, como la física, parecen poco susceptibles de valoraciones sociales, políticas o ideológicas ya que están muy alejadas de lo humano y lo social, mientras que otras, como la biológica 0 la social, lo son bastante más. Es decir, la naturaleza del objeto de estudio determinaría el grado de influencia de lo externo. La filosofía ha de establecer en cada caso cuál es ese grado, por eso es tan importante en su propuesta el no tratar globalmente a la ciencia y proceder a través del examen de casos y contextos de investigación.

Por tanto, según Longino, hemos de aceptar que la ciencia es lo que es, compleja, y resultado de procesos internos e influencias contextuales. La única alternativa factible es hacer explícitos los valores desde los que se está investigando para reconocerlos y saber en cada caso donde estamos, haciéndonos conscientes de su existencia. La objetividad tiene que ver con esta estrategia no con el objetivismo de signo positivista que implica la descontaminación valorativa de la cien$\mathrm{cia}^{14}$.En lo que se refiere a la cuestión de los sesgos de género, las tesis de Longino son enormemente iluminadoras. Como ella misma muestra en el caso de las ciencias biomédicas, las llamadas ciencias del cuerpo encargadas de establecer los fundamentos científicos de la naturaleza humana, la incidencia de valores androcéntricos y misóginos han sesgado profundamente sus teorias desde sus orígenes.

El trabajo que se desarrolla a continuación se sitúa en el contexto de la propuesta de esta autora y lo que se pretende poner de manifiesto es cuán influidas están las teorias e investigaciones que se llevan a cabo por la ideología androcéntrica dominante, que forma parte de los supuestos de trasfondo que los científicos del momento comparten. En él se llevará a cabo el examen de las disciplinas biomédicas del $\mathrm{S}$. XIX, centrándose en el análisis de un episodio concreto de gran importancia en la conceptualización de la naturaleza femenina moderna: los estudios del cerebro y, especialmente, la teoría de la inferioridad mental de la mujer tal como fue formulada por P. J. Moebius a finales del siglo XIX.

\section{Las ciencias decimonónicas y el uproblema de la mujern}

En el siglo XIX la ciencia se entendia como el discurso de la verdad, la más autorizada palabra sobre cuestiones natura- 
les, sociales, e incluso, humanas. Su prestigio era enorme, era considerada como la forma de conocimiento más auténtica, mejor y más efectiva, prominente respecto a la teología y la metafísica. Ella ofrecia un acercamiento desapasionado, calmado y objetivo a las grandes cuestiones que tenía planteada la humanidad. La pregunta acerca de la naturaleza humana era una de estas cuestiones.

El interés de la época por estudiar a la humanidad y hacerlo en términos científicos fue enorme. Se entendió, como ya habian señalado diversos pensadores del siglo anterior, que la ciencia permitiria, alcanzar un conocimiento auténtico, objetivo y verdadero de lo humano y lo social, igual que habia hecho en otros campos. La necesidad de una ciencia de los seres humanos (razas, sexos, culturas y grupos humanos), que diera cuenta de sus especificidades, diferencias naturales y de la función que correspondia a cada uno en el nuevo orden social, se convirtió en algo urgente. Las disciplinas encargadas de llevar a cabo este estudio fueron las nuevas ciencias biológicas, médicas y sociales que se constituyeron como tratados de la diferencia entre sexos, razas, clases, culturas y pueblos. Estas disciplinas comenzaron a desarrollarse, en el s. XVIII, como historia natural descriptiva y como fisiología, estableciendo amplias taxonomías para crear un estudio comprensivo de los organismos vivos, pero también de los seres humanos y los pueblos. El patrón que se sigue en el caso del estudio de nuestra especie es el mismo que se aplica al estudio de los organismos vivos siguiendo un modelo naturalista de comparación y diferenciación entre razas, sexos, culturas, grupos.

La cuestión de las diferencias entre hombres y mujeres, y el papel que correspondía a cada sexo en el orden social fue un tema de gran interés científico. El que se conoció como problema de la mujer ocupó a especialistas de los distintos campos del conocimiento biomédico y social del momento. El supuesto básico que operó como trasfondo de las investigaciones fue la creencia de que hombres y mujeres se diferenciaban fisiológica, anatómica, morfológica y funcionalmente $y$, por tanto, sus facultades, capacidades y habilidades eran muy distintas. Ambos sexos fueron concebidos como dos cuerpos diferentes con dos naturalezas opuestas. Se instaura una concepción de los sexos que afirma el dimorfismo radical y la divergencia biológica entre ellos. Las nuevas ciencias establecen una anatomía y una fisiología de lo inconmensurable que sustituye a la metafísica de las jerarquias en la representación de los seres humanos. Las mujeres pasan a ser consideradas, no como una versión menor del hombre en un eje vertical de infinitas gradaciones, sino como una criatura completamente diferente a lo largo de un eje horizontal cuya parte intermedia estaba totalmente vacia ${ }^{15}$. La fisiología, la anatomía, la frenologia, la neurología, la antropología física y la psicología diferencial se ocuparon de detallar tales diferencias de forma pormenorizada. Desde cada uno de ellas se establecieron todo tipo de hechos diferenciales encaminados a mostrar la inferioridad natural de las mujeres respecto a los hombres (blancos, europeos). Así, las disciplinas biológicas, médicas y sociales se convirtieron en el fundamento último de las afirmaciones normativas sobre las mujeres y su lugar en la sociedad dada su naturaleza.

La aparición de la Teoria de la Evolución en la segunda mitad del siglo con la publicación en 1859 del Origen de las especies de Darwin, proporcionó fundamentación teórica, desde principios generales, a todas las observaciones que se habian establecido anteriormente. Su efecto fue revolucionario y su influencia absoluta dando un mayor alcance a los hechos particulares de la anatomía, la fisiología, la frenología, la antropología y la psicología. Los evolucionistas del XIX se ocuparon reiteradamente del problema de la mujer. Darwin fue el primero que dio pie a ello a través de su famoso trabajo, La descendencia del hombre y la selección en relación al sexo de 1871, dos volúmenes dedicados al estudio minuciosamente detallado de las diferencias sexuales de los animales según una linea evolutiva progresiva ${ }^{16}$. Los trabajos de Spencer ${ }^{17}$ (con resonancias lamarckianas), y los de evolucionistas como Frank Ferneseed ${ }^{18}$ o G. Ferrero ${ }^{19}$ abordaron directamente la cuestión de la inferioridad evolutiva femenina en términos de selección natural y sexual, ofreciendo una explicación evolucionista de las diferencias encontradas entre hombres y mujeres. Durante lo que queda de siglo las inferiores características de las mujeres fueron consideradas innatas y evolutivamente configuradas, oponiéndose a las tesis de S. Mill, quien habia señalado la importancia de la situación subordinada de la mujer y de su imposibilidad de acceder a la educación ${ }^{20}$.

Con el evolucionismo, la naturalización de la inferioridad femenina encuentra fundamento teórico ${ }^{21}$. Las mujeres, igual que otras razas y pueblos repiten ,en su propia historia, la de la evolución de la especie. En la ontogénesis la mujer representaba la adolescencia eterna, estaba cercana al niño, en la filogénesis recordaba el antepasado de la raza, el salvaje, y ambos eran eslabones inferiores en la gran cadena de la evolución. La relación de los hombres civiliza- 


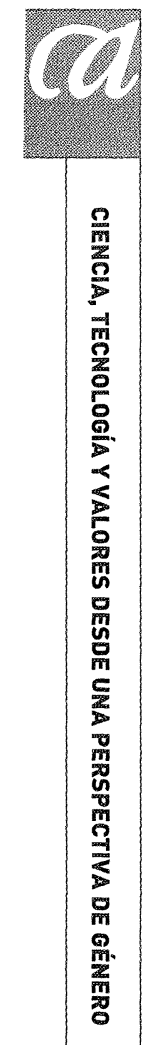

dos con salvajes y mujeres era como la de un padre con su hijo, estableciéndose un paralelismo natural entre niño, primitivo y mujer ${ }^{22}$. Los evolucionistas estuvieron de acuerdo en que la mujer había tocado techo evolutivo y no seguiria evolucionando dadas sus funciones naturales. Herbert Spencer fue quien desarrolló esta tesis que fue generalmente aceptada. Detengámonos un momento en los argumentos de este autor.

En su artículo La psicología de los sexos 23 , Spencer argumentó que los hombres y las mujeres eran tan desiguales mentalmente como lo eran fisicamente. Afirmar lo contrario sería "suponer que sólo en este caso en toda la naturaleza no hay un ajuste de poderes especiales a funciones especiales. La función de las mujeres es criar a los hijos, los atributos intelectuales no son necesarios para esta tarea, por tanto, no tienen por qué desarrollarse en el curso de la evolución" 24 . De esta manera, Spencer, partiendo del supuesto de que la función de las mujeres se reduce a procrear y criar, y que tal función no exige cualidades de orden superior, concluye que las cualidades superiores no tienen por qué ser desarrolladas por ellas. Aún cuando no se cuestionaran las premisas, que obviamente son totalmente cuestionables, en el argumento de Spencer encontramos un paso inaceptable de lo que supuestamente es a lo que debe ser. Todo lo que se puede concluir de las premisas propuestas es que la mujer no tiene cualidades intelectuales superiores. Si se concluye que no sólo no las tiene, sino que no tiene por qué tenerlas, o lo que es lo mismo, no debe tenerlas, es porque en el razonamiento existe una premisa velada, implícita: que las mujeres podrian llegar a adquirir tales cualidades de alguna manera.

Esto es lo que Spencer vio y trató de contrargumentar introduciendo en el seno de la teoría una dirección interesada. En sus propias palabras sostiene que «bajo disciplinas especiales, el intelecto femenino podria igualar o sobrepasar los resultados intelectuales de la mayoría de los hombres» 25 . ¿Cuál es entonces el problema?, ¿por qué no dejar que las cosas sigan su curso natural y las mujeres evolucionen cambiando sus condiciones de vida? La respuesta de Spencer fue contundente: porque entonces tales cualidades "disminuirian totalmente las funciones maternales", irian en detrimento de ellas. A la pregunta acerca de por qué es esto posible Spencer contesta: porque las mujeres "necesitan energía para cumplir sus funciones de madre y si esa energía se gasta en otras tareas se la está restando de la que es la única función natural de la mujer» 26 .
Esta afirmación se basaba en un principio científico comúnmente aceptado en ese momento. Se creia que el cuerpo humano contenía una cantidad limitada de energía o fuerza vital y que este recurso podía agotarse si se le utilizaba mal. La tesis se basaba en la idea de que la energía puede disminuir por su uso en el trabajo y se convierte en paradigma en los estudios del metabolismo animal. El cuerpo se ve como una máquina con su propia energía que produce calor, es como una locomotora viva guiada por el cerebro. La energia psíquica es fundamental, la salud depende de la moderación del gasto de la energía, y el agotamiento nervioso es la peor amenaza. Esta idea fue central en la argumentación de los evolucionistas y de los médicos en contra del trabajo físico e intelectual de las mujeres. Agotamiento físico, desarreglos, esterilidad y enfermedad mental aquejará a las mujeres que gasten su energía en funciones que no son propias de su naturaleza, no pueden soportar el desgaste de la maternidad y el que supone esas otras actividades. La reproducción es un sistema delicado que implica un extraordinario gasto de energía, la menstruación además debilita y enferma a las mujeres. Por tanto, no deben tener acceso a la educación superior ni al trabajo. El cuerpo humano, "no puede hacer bien dos cosas al mismo tiempo". Las chicas adolescentes necesitan reducir su trabajo cerebral en los años del desarrollo reproductivo, su energía no debe invertirse en la escuela como los chicos cuya naturaleza les pide menos. Si se transgrede esto se pierde en salud, se enferman; la lucha entre lo que pide el cerebro para su educación y la reproducción acaba con ellas. Si hacen mal uso de su vida, sus descendientes se resentirán. Por otro lado, no tiene sentido permitir el acceso de las mujeres a la educación superior y permitirles acceder a actividades y cargos de poder y responsabilidad puesto que, a lo anterior, hay que sumar su menor capacidad intelectual 27. Médicos y evolucionistas coincidian en afirmar que la energia femenina era la única que podía coexistir con la producción y crianza del debido número de niños sanos. Por tanto, las mujeres debian administrarla bien, so pena de generar terribles consecuencias para la evolución de la especie y la sociedad. Spencer afirma, "si las mujeres desarrollaran cualidades mentales en una sociedad provocarian la desaparición de la sociedad, es un poder que no debe incluirse en una estimación de la naturaleza femenina" 28 . Por tanto, en cuanto las mujeres dejaran de emplear toda su energía en la que era su función natural (la procreación), la especie se resentiria, dege- 
neraría y perdería parte de sus más altos logros evolutivos.

Por qué esto es así y el futuro de la especie proyecta tan terrible responsabilidad sobre las mujeres es algo que Spencer explica desde otro postulado del momento: la creencia de que la mayor parte de los rasgos físicos, mentales $e_{\text {, }}$ incluso morales, pasan directamente de padres a hijos, siendo heredados tal cual por estos. De esta manera si las mujeres degeneran física o mentalmente por el mal uso de su fuerza vital, su degeneración se trasmitirá directamente a sus hijos y descendencia futura ${ }^{29}$. Deben permanecer tal como son, su naturaleza ha de subordinarse a las exigencias de la Naturaleza; la especie las atrapa, como evidencia el mismo Spencer cuando afirma que las mujeres son, sencillamente, un caso de desarrollo evolutivo arrestado. No necesitan seguir evolucionando para cumplir sus funciones, han tocado techo evolutivo. La evolución de las mujeres es combatida por el interés de la especie, las mujeres están al servicio de la especie, es su mejor instrumento y no deben cambiar. Están condenadas a repetir el ciclo necesario de la vida. Por decirlo con palabras de Simone de Beauvoir: la mujer no puede hurtarse al dominio de la especie, se subordina a ella 30 .

Es precisamente porque deben reservar su energía, dado el gran costo de la reproducción, que las mujeres tienen un menor desarrollo muscular y nervioso $y$, por tanto, una menor capacidad para las manifestaciones mentales. "Son un colectivo disminuido en aquellas facultades intelectuales y emocionales máximos productos de la evolución humana, el poder de razonamiento abstracto y de la más abstracta de las emociones, la justicia» 31 . Esto explica, por qué las mujeres no son capaces de hacer juicios que vayan más allá de lo personal y por qué se ponen obvias objeciones a extender a ellas ciertos privilegios como el poder de legislar o dictar sobre injurias. La argumentación se ha desplazado hacia el terreno de la moral en el cual las mujeres son, también, evolutivamente inferiores.

Este tipo de argumentación la encontramos, prácticamente en los mismos términos, en el trabajo de Moebius sobre la inferioridad mental de las mujeres, lo que muestra la influencia del evolucionismo sobre las investigaciones del cerebro. Aunque al mismo tiempo los evolucionistas se apoyaron en los datos que ofrecian estas investigaciones que interpretaron como confirmación de los argumentos evolucionistas acerca de la inferioridad femenina. Los estudios del cerebro fueron, a lo largo de todo el siglo XIX, un recurso fundamental en la demostración científica de la inferioridad de las mujeres y sus resultados fueron ampliamente aceptados por la comunidad científica.

\section{Los estudios del cerebro}

Los estudios del cerebro se desarrollaron desde finales del siglo XVIII y a lo largo del XIX sobre todo por frenólogos, neurólogos y antropólogos físicos y fueron claves en la constitución del discurso "científico" acerca de la inferioridad de las mujeres (también de las razas no blancas, las culturas y pueblos no civilizados y clases sociales más bajas). La frenología, cuyos comienzos estuvieron en los trabajos de Franz J. Gall, marcó el inicio de la investigación "científica» del cerebro. Franz Joseph Gall ${ }^{32}$ estableció el estudio de las bases materiales de los rasgos psicológicos. El consideró que había una correlación positiva entre memoria, y otras facultades mentales, y la conformación del cráneo. Estudió la configuración del cráneo entendiéndola como indicativa de facultades mentales y rasgos de carácter, afirmando que las caracteristicas mentales de un individuo podian determinarse examinando las formas e irregularidades de su cráneo. La "lectura de cabezas" disfrutó de popularidad durante tiempo ${ }^{33}$.

Gall no duda de que el cerebro es el órgano de la mente, de que la mente estaba constituida por facultades independientes, de que estas facultades eran innatas y de que cada una de ellas estaba situada en una región de la superficie del cerebro. Sostiene que el tamaño y forma de cada región craneal es la medida del grado en el que la facultad correspondiente se ha desarrollado en el individuo. Hay, por tanto, una relación entre la superficie y contorno del cráneo y lo que éste esconde capaz de permitir al observador reconocer la potencia de estas facultades por el examen de tal superficie ${ }^{34}$. Aplicó su nueva ciencia al estudio de las peculiaridades craneales que presentaban los enfermos mentales o criminales (frente poco desarrollada, protuberancias, etc.), lo que le permitió inferir que las personas con esos rasgos eran enfermos o criminales potenciales (fue un determinista fisiológico).

Los trabajos de Gall fueron divulgados por su discipulo J. G. Spurzheim ${ }^{35}$ y la nueva ciencia de la frenologia se hizo popular. Éste mantenia que habia una diferencia natural en las disposiciones mentales de hombres y mujeres, en cuali-

ARBOR CLXXXI 716 NOVIEMBRE-DICIEMBRE (2005) 479-492 ISSN: 0210-1963 
dad y cantidad, que la educación no puede cambiar. Afirmaba que ciertos poderes mentales eran más fuertes en el hombre que en las mujeres, el intelecto de éstas tenía menor vigor y un poder reflexivo más pequeño, y que las mujeres y los negros no extienden su razonamiento más allá del mundo visible. En el hombre predomina el intelecto sobre el sentimiento, en la mujer es al revés.

Los estudios frenológicos culminaron con el establecimiento del indice cefálico (relación entre anchura y longitud del cráneo) por Anders Retzius en 1840. El índice cefálico, junto al peso del cerebro, se convirtió en la piedra angular de la antropometría para todo lo que quedaba de siglo. Este índice fue considerado por los antropólogos como indicador sumamente significativo de diferencias fundamentales en los rasgos mentales, actitudinales, comportamentales de las razas, los sexos y otros colectivos humanos. Tales opiniones se aceptaron durante mucho tiempo como científicamente sólidas, fueron centrales en los estudios antropológicos y psicológicos y sirvieron de apoyo a la idea de que los conflictos incesantes entre los pueblos podian explicarse basándose en las diferencias cerebrales.

Los antropólogos destacaron el mayor tamaño y peso del cerebro de los hombres de raza blanca y el menor peso, volumen e índice cefálico del de las mujeres (otros pueblos y razas). Los datos sobre las diferencias cerebrales entre hombres y mujeres se acumularon. Paul Broca examinó 500 cerebros (de diferentes razas y sexos) y tomó sobre ellos más de 180.000 medidas. Vogt pesó 2086 cerebros masculinos y 1061 femeninos. Todo ello vino a demostrar que en el caso de las mujeres su cerebro pesaba entre 113 y 140 gramos menos que el de los hombres (estos tenian una ventaja de un 10\% de volumen y peso). La cuestión de las cinco onzas perdidas del cerebro femenino se hizo popular en Inglaterra. Estos fueron considerados datos de enorme trascendencia y de ellos se infirió que las mujeres eran inferiores en sus capacidades y facultades mentales, intelectuales y morales, y en todo lo que dependia de aquellas.

Broca ${ }^{36}$, confirmó que las facultades mentales estaban situadas en órganos cerebrales especificos al investigar los desórdenes lingüisticos producidos por lesiones cerebrales. Fundó la Sociedad Antropológica de Paris que se convirtió en el gran centro europeo de tipología racial y modelo de otras sociedades similares que se fueron extendiendo en Londres y América. El consideró que raza y sexo eran dos caras de la misma moneda. Las mujeres, igual que las razas inferiores tenían un menor índice cefálico y su cerebro era menos pesado, estaba más cerca del de los negros que del de los hombres blancos, sus cualidades mentales se asemejaban ${ }^{37}$.Carl Vogt, profesor de historia natural de Génova con intereses antropológicos, enfatizó la diferencia y especificidad tanto sexual como racial del cerebro. En su obra Lectures of Man afirmó que los cráneos de hombres y mujeres podian ser separados como si pertenecieran a dos especies diferentes ${ }^{38}$. James McGrigor Allan ${ }^{39}$ consideró que el tipo de cráneo de la mujer se parecia en muchos aspectos al del niño, y todavía más al de las razas inferiores. Mantuvo que el cerebro de los hombres más primitivos y el de las mujeres eran casi iguales en tamaño, peso y, por tanto, facultades. El de los hombres blancos civilizados era superior en todos estos aspectos. La teoria de la evolución apuntala estas investigaciones al explicar que la inferioridad de la mujer (y otros grupos humanos) respecto al hombre blanco, se debía a que sus estadios evolutivos son diferentes, pues el cerebro de las mujeres está menos evolucionado y es más inmaduro.

Sin embargo, a finales del siglo XIX la frenología estaba desacreditada, la cuestión del volumen y peso del cerebro ya no era aceptada como relevante (se relaciona el cuerpo en que está el cerebro con su peso y volumen entendiendo a éstos en términos relativos), se cuestiona el concepto de localización cerebral y se plantea el tema de la unidad funcional del cerebro ${ }^{40}$. A pesar de todo esto no se abandona el estudio de las diferencias cerebrales entre los sexos que, a finales del XIX, se plantean como diferencias estructurales y cualitativas. Aparece la idea del mejor material y la construcción cerebral mejor y más elaborada. En este contexto aparece en 1900 la obrita de Moebius La inferioridad mental de la Mujer ${ }^{41}$.

\section{La inferioridad mental de la mujer}

Moebius vivió entre 1853 y1907, estudió teología y medicina, vástago de una dinastía de científicos, se dedicó a la neurología en el Policlínico Universitario de Leipzig y en el Policlínico Neurológico de Albert-Verein. Simpatizó con la frenología, aceptó plenamente la teoría de la evolución y fue un gran un admirador del pensamiento de Schopenhauer. Todo ello le convierte en prototipo de científico acreditado en la época.

Moebius compartió la idea, que trató de demostrar con su trabajo, de que las diferencias morfológicas en el cerebro de 
hombres y mujeres eran claves para explicar la inferioridad de éstas. Basándose en las investigaciones del anatomista Rüdinger sobre tales diferencias elaboró una propuesta, que condensó en unas veinte páginas, en la que pretendió demostrar científicamente la inferioridad mental de las mujeres ${ }^{42}$. Los hechos diferenciales en cuestión eran, en palabras de Moebius : que "Rüdinger ha observado que en los recién nacidos el grupo de circunvoluciones en torno a la cisura de Silvio es más sencillo y posee menos sinuosidades en las hembras que en los machos; además, que la isla de Reil es, en término medio, un poco mayor en todos sus diámetros, más convexa, está surcada en el cerebro de los varones más profundamente que en el de las hembras. $\mathrm{Ha}$ demostrado que en los adultos la tercera circunvolución frontal es más pequeña en la mujer que en el hombre, especialmente en aquellas secciones que lindan directamente con la circunvolución central... Además, Rüdinger ha probado que en el cerebro femenino el derrame de toda la circunvolución media del lóbulo parietal y la del pasaje superior superointerno presentan un desarrollo insuficiente» 43 . Como colofón a estos datos Moebius afirma:" En todos sentidos queda completamente demostrado que: en la mujer están menos desarrolladas ciertas porciones del cerebro de suma importancia para la vida mental, tales como las circunvoluciones del lóbulo frontal y temporal; $y$ que esta diferencia existe desde el nacimienton ${ }^{44}$.

Moebius consideró que las observaciones de Rüdinger, no tan conocidas como las realizadas por los frenólogos, "tienen especial importancian ${ }^{45}$. El las toma como base cientifica de su argumentación dándolas por sentadas y sin indicar nada acerca de la investigación llevada a cabo, ni los procedimientos científicos seguidos por Rüdinger. Señala únicamente que es un estudio comparativo y que los datos son más fiables que los establecidos en relación al tamaño del cráneo o el peso del cerebro.

Que la investigación de Rüdinger se basara en un estudio comparativo plantea una interesante cuestión respecto a la fiabilidad de los resultados que Moebius hace suyos. Las observaciones que Rüdinger llevó a cabo se hicieron sobre cerebros diferenciados y clasificados sexualmente (cerebros de hombres y mujeres) objeto de comparación. Pero esta distinción inicial no se limita a dividir el universo objeto de estudio en dos ámbitos neutramente distinguidos sobre los que se establece observaciones e inferencias desprejuiciadas. Lo que se hace es separar cerebros "masculinos" y "femeninos" y esta distinción incorpora los supuestos pro- pios de cada género. Lo que se observa comparativamente son cerebros de hombres altamente evolucionados $y$, por tanto, dotados de cualidades superiores y cerebros de mujeres menos evolucionados $y$, por tanto inferiores. Es decir, la diferenciación entre cerebros de uno y otro sexo nos deja con unos hechos observacionales que ya incluyen el prejuicio (el supuesto) de la inferioridad femenina (lo mismo ocurre con los estudios sobre otras razas). Las observaciones se limitarán a manifestar lo que está implícito en la misma muestra observacional: que hay hechos diferenciales y que estos muestran la inferioridad de las mujeres. Cualquier dato diferencial que se encuentre será interpretado como indicativo de la inferioridad supuesta. La interpretación de las diferencias morfológicas viene determinada por el a priori de la superioridad masculina que contamina todas las inferencias que se puedan establecer. La norma respecto a la cual se valora negativamente cualquier diferencia es la morfología del cerebro de los hombres blancos, todo lo que se desvie de ella es interpretado como inferior. La diferencia es siempre de los otros y siempre negativa: "mujeres", "salvajes", uidiotas", "negros". Cómo señala Longino, la injerencia no sólo está cargada de teoría sino que además da por sentado unos supuestos de base y unos valores contextuales que la sesga profundamente.

En el caso de Moebius la cuestión que se pretendia demostrar era clara, pues, antes de entrar en consideraciones sobre Rüdinger afirma: "Es indudable que las facultades mentales del hombre y de la mujer son muy diferentes entre sí... ¿pero son las mujeres verdaderamente deficientes respecto a los hombres?» ${ }^{46}$. Respondiendo inmediatamente: "Un antiguo proverbio da la respuesta: Cabellos largos, cerebro corton 47 , y los argumentos ofrecidos por diversos autores del momento "demuestran la inferioridad mental de la mujern ${ }^{48}$. Lo que él propone es "considerar también los datos anatómicos" para confirmar la deficiencia de la mujer, lo que le lleva a las investigaciones de Rüdinger ${ }^{49}$. Por tanto, primero se da el supuesto de la deficiencia de las mujeres y, luego, su demostración científica por recurso a la naturaleza femenina. Tal como él plantea la cuestión, el interrogante que guia su estudio está contestado a priori, la argumentación que desarrolla a lo largo de su trabajo puede considerarse mera retórica que justifica lo que ya se ha dado por sentado. Veamos en qué términos trascurre su argumentación.

A partir de las diferencias morfológicas señaladas por Rüdinger, Moebius prosigue infiriendo dos postulados e introduciendo una noción clave en su análisis, la de instinto. Ambas

ARBOR CLXXXI 716 NOVIEMBRE-DICIEMBRE (2005) 479-492 ISSN: 0210-1963 
cuestiones no se justifican en el recurso a la investigación cientifica, como era de esperar, sino en la evidencia cotidiana que Moebius interpreta en términos claramente negativos para las mujeres, y en supuestos y valores claramente misóginos. Su argumentación se aparta enseguida de las consideraciones anatómicas que, en sus propias palabras, iban a ser centrales, para prestar atención a las manifestaciones psicológicas, morales y del comportamiento que, según el autor, domina en las mujeres ${ }^{50}$.

Los postulados que establece Moebius son:

1. Que las mujeres «tienen una menor reacción psíquica a los estímulos más fuertes» ${ }^{51}$. Sus sentidos no son deficientes pero si su psique de la cual dependen aquellos. Esto lo demuestra el hecho cotidiano de que los hombres son los catadores de vino, aceite, los que prueban el te, seleccionan el algodón...

2. Que la mujer es inferior en cuanto a fuerza y destreza. La habilidad manual es una función de la corteza cerebral; también la valoración de las sensaciones, su corteza cerebral está disminuida por eso su habilidad es menor. Por ello los hombres pueden realizar mejor cualquier habilidad femenina, cocinar, coser, tejer, etc.; siempre producen mejores obras que la mujer ${ }^{52}$.

A continuación Moebius introduce la que considera una de las diferencias esenciales de la mujer «el hecho de que el instinto desempeña un papel más importante en la mujer que en el hombrem 53 . A partir de ello trata de establecer "una línea recta desde los seres que obran por instinto a los seres cuyas acciones están subordinadas a la reflexión „ 54 .

El concepto de instinto le sirve al autor para abrir una línea de argumentación que redunda en la cuestión a demostrar. Este concepto no se deriva de nada de lo afirmado anteriormente y, por tanto, opera como primitivo en su teoría. É lo define afirmando que: " hablamos de instinto cuando se ejecuta una acción coordinada y útil, sin que el que la lleva a cabo sepa el porqué;....cuando acertamos a dar una opinión sin saber cómo" 55 . Dado que el instinto "desempeña un papel más importante en la mujern (premisa que no se considera obligado a probar), su conocimiento es meramente instintivo. $Y$ concluye "El instinto presenta grandes ventajas, es fiable y no proporciona ningún género de preocupaciones. De modo que el instinto hace a la mujer seme- da esa fuerza singular y la hace aparecer verdaderamente admirable $y$ atractivan 56 .

Su tratamiento del instinto le permite mostrar la auténtica naturaleza de las mujeres $y$, al mismo tiempo, recurriendo a una conocida tesis, situarlas en el lugar que les es propio: su mayor cercanía a la naturaleza. El instinto es algo propio de los animales, la parte de la especie humana que aún sigue rigiéndose por él, en lugar de por las facultades superiores, está más cerca de las bestias y, por tanto, en un estadio intermedio de la evolución. De hecho "la característica de un alto desarrollo psíquico está en que el instinto va perdiendo su importancia mientras crece la de la reflexión, en que el ser ligado a la especie se va individualizando cada vez ması ${ }^{57}$. Esto es lo que encontramos en el caso de los hombres pero no en el de las mujeres.

Al partir Moebius de la creencia de que el instinto domina en las mujeres, concluye que éstas carecen del poder de reflexión y de individuación que es propio de los hombres. No han logrado constituirse en individuos desligados de la especie, están en un estadio intermedio entre el hombre y las bestias y sometidas a sus funciones naturales. El progreso de la especie se manifiesta a través de los varones ya que "Como los animales que obran de la misma manera desde tiempos inmemoriales, el ser humano se hallaría estancado en un estado original si no existieran más que mujeres. Todo progreso parte del hombre» 58 .

El símil con la naturaleza proporciona a Moebius argumentos que ratifican la inferioridad de las mujeres. Su argumentación se basa en hechos, que él considera claramente observables, de la psicologia e incluso moral de las mujeres. En sus propias palabras "Muchas caracteristicas femeniles dependen de esta semejanza con la bestia. Ante todo la carencia de opinión propia. Lo que es considerado generalmente bueno y verdadero, es para las mujeres verdadero y bueno. Son rigidas conservadoras y odian la novedad....159. En lo que respecta a la moral, la mujer está entre los animales que se mueven por instinto y el hombre que se ha liberado de él con una capacidad de raciocinio certera; está en el estadio del sentimiento.

De nuevo nos encontramos con un tema recurrente en la concepción dominante de lo femenino y presente en toda la literatura acerca de la inferioridad natural de las mujeres, la cuestión del sentimiento que Moebius define en los siguientes términos: "llamamos sentimiento a un estado 
intermedio entre lo instintivo y lo consciente o sabido" 60 . Definición muy útil puesto que permite apostillar, ahora en el terreno del carácter y la moral, la menor evolución femenina y su carencia de facultades superiores. Moebius afirma al respecto: "las mujeres se detienen en un estado intermedio. Su moral es, sobre todo, una moral sentimental, una rectitud inconsciente" ${ }^{61}$. No son inmorales pero sólo tienen una moral "unilateral o defectuosa", no tienen sentido de lo justo y en el fondo se rien de la ley, "La justicia sin consideraciones personales es para ellas un concepto vacio de sentido" 62 . Por ello las mujeres aparecen adornadas con vicios caracteristicos de su sexo, según el autor, como su gran capacidad de mentir, de ser astutas y ladinas.

El déficit moral es correlativo al intelectual y ambos obedecen a la mayor cercanía de las mujeres a la naturaleza y, por tanto, su limitada evolución. El problema de las mujeres, es sobre todo, la "naturaleza» de la naturaleza femenina. Moebius es claro al respecto, las mujeres están cognitiva y moralmente peor dotadas, pero sobre todo están determinadas por su función natural. Por eso afirma que uel sexo femenino aprende muy poco y en breve olvida lo que ha aprendido" 63 . Sobre todo, carece de las capacidades que exige la actividad intelectual en los diversos campos del conocimiento, como mucho puede repetir monótonamente lo aprendido pero son incapaces de crear, inventar como muestra la historia del pensamiento ${ }^{64}$. La aportación de las mujeres a los grandes logros del pensamiento, la ciencia y las artes es nula, carecen de capacidad para "combinar", de "fantasia" y de un "pensamiento independiente" 65 , a lo que se agrega "una falta de objetividad que las hace transformar los deseos en motivos y las simpatias en pruebas" ${ }^{66}$. Su incapacidad creadora es tan general que uincluso el arte culinario y el de vestir sólo progresan gracias a los hombres, puesto que son ellos los que inventan...Todo cuanto vemos a nuestro alrededor, todos los utensilios domésticos, los instrumentos de uso diario, todo ha sido inventado por los hombres" 67 . Por eso se entiende que "la mujer no ha aportado nada al desarrollo de la ciencia y que resulta inútil esperar algo de ella en el porvenir» 68 .

Sin embargo, estos hechos no deben atribuirse sólo a su "poca capacidad, sino a la falta de voluntad» 69 . Esta falta de voluntad, es sobre todo un déficit de su naturaleza, obedece a que los intereses de las mujeres son uexclusivamente personales". Esto hace que no vean en la instrucción una ventaja personal y upor lo general es contraria a la instrucción ${ }^{70}$. La cuestión fundamental es que las mujeres tienen unos intereses muy marcados por lo que es su fin natural, la reproducción, que exige de ellas "hacerse deseables» 71 . Este "hacerse deseables" es contrario a las cualidades propias de la esfera intelectual como la independencia, el mantenimiento de la propia opinión. Para gustar al otro sexo y cumplir su fin reproductivo las mujeres están dotadas «por naturalezan de cualidades específicas de su sexo, "ella callará todo lo que pueda resultar adverso a la opinión de los demás", se ejercita "en el disimulo" y "la mentira"72. Por tanto, las mujeres no deben dedicarse a aquello para lo que no están dotadas y que les perjudica y, además, es necesario protegerlas de ello en favor de su función natural, afirma: "la hembra debe ser, ante todo madre. Asi en el campo intelectual debemos dar a la mujer todo lo que aligera su tarea de madre y eliminar todo lo que pueda obstaculizarla "73. Por eso, "si queremos una mujer que pueda cumplir bien sus deberes maternales, es indispensable que no posea un cerebro masculino» ${ }^{74}$. El desarrollo de las facultades intelectuales es algo peligroso como está mostrando el hecho de que "la procreación disminuye proporcionalmente al desarrollo de la civilización" ${ }^{75}$. Y esto "hace degenerar a la raza" y urepresenta el principio del fin" ${ }^{76}$. Por eso no debe dárseles instrucción como creen algunos pensadores "que han sugerido a las mujeres la manía de la emancipación” 77 .

Las mujeres no pueden participar ni de la educación, ni de la esfera pública en general, dado el déficit intelectual y moral intrínseco a su sexo por naturaleza. Esta naturaleza deficitaria es, sin embargo, necesaria para cumplir con sus funciones naturales, la procreación y " la necesidad de cuidar a los niños». El que "la hembra humana no sólo deba parir los hijos, sino también cuidarlos.... es la causa de que la diferencia entre los sexos sea más marcada en la especie humana que en las especies animalesin 78 .

Moebius se pregunta "cómo debe estar constituida esta naturaleza para realizar del mejor modo posible la misión que le ha sido encomendada?» ${ }^{79}$. La respuesta es: tal como está evolutivamente constituida la naturaleza femenina; o lo que es lo mismo, en los términos en que Moebius acaba de describirla. Por tanto, "la deficiencia mental de la mujer no sólo existe sino que se hace muy necesaria; no solamente es un hecho fisiológico, es también una exigencia psicológican para la especie ${ }^{80}$. Y afirma, citando a Lombroso, que está demostrado que uen todo el reino animal la inteligencia se halla en razón inversa a la procreación ${ }^{81}$. La función natural de las mujeres les impiden desarrollar facultades 
superiores, la especie no necesita de ellas para perpetuarse. Ir en contra de esto es ir contra natura. Moebius es nítido al respecto, "Yo creo que el punto más importante para los médicos es que ellos se formen un claro concepto del cerebro o del estado mental de la mujer, y que comprendan bien el significado y el valor de su deficiencia mental; y que hagan todo lo posible para combatir, en interés del género humano, las aspiraciones contra natura de las feministas. Se trata de la salud del pueblo, en peligro por la perversión de la mujer moderna. La naturaleza es un amo inflexible y castiga con penas severas a los infractores de sus leyes» ${ }^{82}$. El argumento, y la forma de expresarlo, son prácticamente idénticos a los utilizados por Spencer y los evolucionistas. A partir de aqui el autor se dedica a lanzar diatribas contra las feminista y la perversión de la mujer moderna afirmando que la naturaleza castiga a los que pretenden escapar a sus dictámenes y eso es lo que pasa con aquellas que quieren huir de su destino, se masculinizan y degeneran.

No hay que olvidar el contexto social y político en que que se desarrolla el trabajo de Moebius ${ }^{83}$. A partir de la segunda mitad del siglo XIX las mujeres entran masivamente en el mercado de trabajo, más explotadas que los hombres comienzan a luchar por la igualdad salarial y el acceso a la instrucción, por el sufragio, etc. Stuart Mill, las sufragistas, las luchas de obreras, las ligas de braceras, voces femeninas diversas, incitan a la lucha y a la conquista de los derechos negados siglo tras siglo. Una nueva conciencia política se está formando entre las mujeres. Este es el contexto en el que se insertan las voces de Moebius y Lombroso que utilizan la ciencia para oponerse a la insubordinación de la mujer.

De esta manera termina de articularse una historia que comienza haciendo referencias puntuales a diferencias morfológicas (que no ocupan apenas espacio en la argumentación) para ir desgranando todos los tópicos dominantes en el XIX acerca de las mujeres engarzados en una vaga retórica científista.

\section{Consideraciones finales}

La teoria de la inferioridad mental de las mujeres es una excelente muestra de cuan profundamente sesgadas e ideológicamente contaminadas pueden estar las afirmaciones que en nombre de la ciencia, y desde ella, se han llevado a cabo (lo que ya se percibió claramente desde el primer momento sien- do la obra de Moebius calificada por algunos de "panfleto", "folleto sensacionalista" etc.) Su teoria es un buen ejemplo de lo señalado por Longino acerca de la importante incidencia que tienen los supuestos de base o de trasfondo y los valores contextuales desde los que se interpreta y conceptualiza los datos (en este caso anatómicos y cotidianos) y se formulan teorias. La distancia entre las diferencias morfológicas y las consecuencias psicológicas, intelectuales, morales y caracteriológicas señaladas por Moebius supone un vacio que el autor llena con ideología. Lo que Moebius, postula como teoría cientifica no es sino una amalgama de ingenuas observaciones, interpretaciones sesgadas, falacias, ideas tradicionales y diatribas en contra de las mujeres. Los resultados a los que llega el autor constituyen una mera sarta de clichés y prejuicios que confirman el postulado de partida acerca de la inferioridad de las mujeres.

La obra de Moebius, que se reeditó enseguida más de ocho veces, dio lugar a un considerable debate en la época. Los escritos a favor y en contra proliferaron; para unos su libro mostraba la auténtica naturaleza de las mujeres, para otros era un mero panfleto ideológico de corte schopenhauriano.

La principal crítica viene muy bien expresada en varios escritos de Klara Müller ${ }^{84}$ y de Hedwing Dohm ${ }^{85}$ quienes ponen el dedo en la llaga al cuestionar el estatus científico de los hechos anatómicos aducidos, el significado de tales hechos para el tema en cuestión y las interpretaciones que Moebius lleva a cabo. Cuestionan su recurso al instinto considerándolo un tópico que "Moebius, por lo demás, ni siquiera intenta comprobar, confiando en que los lectores masculinos sean tan uinstintivamente conservadores y enemigos de lo moderno" 86 . Así mismo rechazan el tratamiento que hace Moebius de la maternidad y la crianza de los niños y recuerdan la situación de las mujeres alejadas de la educación y la vida pública. Señalan la importancia de las investigaciones de los teóricos del medio ambiente que dan cuenta de las condiciones de vida de las mujeres y se sitúan en la línea de reflexión de S. Mill. Moebius responde a estas críticas, en varios prólogos a las sucesivas reediciones de su obra reafirmándose en sus tesis ${ }^{87}$. Por su parte, los escritos a favor de la posición de Moebius celebran que éste haya podido demostrar la inferioridad de las mujeres como un hecho determinado por su propia naturaleza frente al cual nada cabe hacer 88 .

El debate que se refleja en los escritos señalados muestra la relevancia ideológica, social y política del tema. Esto es así 
ya que el cuerpo femenino reducido a hechos diferenciales específicos de la biología y la anatomía se habia convertido en campo de batalla de la definición de la relación social básica entre hombres y mujeres, fundamento del orden social asentado durante el siglo XIX. La argumentación excluyente de la mujer de la esfera pública se había desplazado de la filosofía, la religión o la tradición hacia la ciencia y su recurso a la naturaleza. A lo largo de todo el siglo, la ciencia fue la encargada de zanjar la cuestión mostrando que las mujeres no estaban dotadas para lo público y que su lugar natural estaba en el ámbito de lo privado-reproductivo. Por ello, como señala Dorinda Outram "en todas partes la biologia entra a formar parte del discurso legitimador en contra de la mujeres aportando pruebas de su inadecuación en todos los órdenes para ocupar la esfera pública y admitir los progresos que reclamanı 89 . Los cientificos estaban haciendo algo más que ofrecer datos a los ideólogos, se convirtieron ellos mismos en ideólogos. Fueron los encargados de demostrar que las aptitudes naturales de las personas eran la base de sus roles sociales convirtiéndose a menudo en árbitros sociales como ocurrió con la cuestión de las mujeres. Esta situación se prolongó en el siguiente siglo en relación a varios episodios científicos conocidos, sobre todo en el caso de la neuroendocrinologia, las teorias de la lateralización cerebral, los estudios de la diferenciación sexual del cerebro, de la evolución humana y la sociobiología.

Porque no debemos pensar que la historia ha terminado. La idea de que el cerebro se diferencia sexualmente y que ello determina las capacidades intelectuales de cada sexo se mantiene en el siglo XX. A modo de ejemplo podemos citar las palabras, de J. A. F. Tresguerres, del departamento de Fisiología de la Facultad de Medicina de la Universidad Complutense, quien afirma en un reciente libro: «... la diferenciación sexual cerebral [es]justificativa de los papeles distintos que asumen ambos sexos no solamente en lo que respecta a los papeles sexuales sino también en lo que respecta a las capacidades intelectuales distintas que presenta cada caso" ${ }^{90}$. Las cuantificaciones diferenciales siguen siendo objeto del mismo interés que tuvieron en el XIX. En el terreno de la neuroendocrinología las mediciones hormonales y de elementos internos del cerebro, por ejemplo del área preótica medial (con resultados superiores en hombres que en mujeres: el doble de un milimetro en hombres) siguen constituyendo la base explicativa de actitudes y conductas diferenciadas y marcadas por el sexo: agresividad, instinto maternal, etc. etc. $Y$, a su vez, han sido, y son, claves en la interpretación determinista biológica de la homosexualidad y lesbianismo ${ }^{91}$. 
1 La redacción de este trabajo ha sido facilitada por la concesión, por parte de la DGESIC, del Proyecto de Investigación $n^{\circ}$ PB98-0495-C08-05.

2 E. F. Keller afirma: el enfoque de género "ha dado lugar a una forma de atención, sobre el conocimiento cientifico como una lente que focaliza una cuestión particularn. Keller, E. F. Reflexiones sobre género y ciencia, Valencia, Edicions Alfons el Magnànim, 1991, p. 14.

$3 \mathrm{El}$ análisis de los sesgos de género en ciencia ha sido ampliamente desarrollado y ha dado lugar a una enorme bibliografía. La mención sólo de los trabajos más relevantes supondria un espacio ingente por lo que nos limitamos a dar algunas referencias de nuestro país a modo de ejemplo. Pérez Sedeño, E., "Filosofía de la ciencia y feminismo: intersección y convergencian Isegoría, no 12, 1995, Pérez Sedeño, E., "Mujer y ciencia: una perspectiva", Introducción al monográfico de Arbor, Mujer y ciencia. (1993). Pérez Sedeño, E., "Nuevos retos, nuevas soluciones: Feminismo y CTS", en Interacciones ciencia y Género, Madrid, Akal, 1999. Sánchez, Torres, A., "El debate sobre la selección sexual:complejidad versus determinismon, en Arbor, (1993). Gómez, A. y Perdomo, I., "El eterno femenino:hormonas, cerebro y diferencias sexuales" Arbor, (1993) Gómez, A., "Usos i abusos de la ciència", Quaderns, no 1, (1995). Gómez, A., "¿Es el sujeto feminista epistemológicamente relevante en ciencia?» en Politica y Sociedad, Revista de la Universidad Complutense, Facultad de Ciencias Politicas y Sociología, no 30, Enero-Abril (1999), pp. 23-39. Gómez, A., "El lugar de lo natural en la construcción científica de lo femeninow en Monzón, M. E. \&t Guerra, M. J. Mujeres, espacios y tiempos. Un análisis desde la perspectiva de género. Sta. Cruz de Tenerife; Instituto Canario de la Mujer, 1999, T. Ortiz y G. Becerra (Eds), Mujeres de ciencias: mujer, feminismo y ciencias naturales, experimentales y tecnológicas. Granada, Ed. Universidad de Granada, Instituto de Estudios de la mujer. Barral, M J, Magallón C, Sánchez M.D. (Eds), Interacciones ciencia y género. Barcelona, Icaria.1999.
4 Las principales posiciones son, empirismo clásico, empirismo femenista y epistemologias radicales provenientes del postmaterialismo y el postmodernismo. Para este tema véase Gómez, A., "De la mujer en la ciencia a las epistemologias feministas" en Gómez, A \& Tally, J. (Eds.) La construcción cultural de lo femenino; Sta. Cruz de Tenerife; Instituto Canario de la Mujer Consejeria de Empleo y Asuntos sociales del Gobierno de Canarias. 1998. pp. 211252.

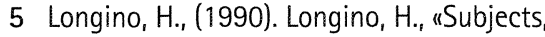
Power and Knowledge: Description and Prescription in Feminist Philosophies of Sciencien en Alcoff, L. and Potter, E. (ed) Feminist Epistemologies, N. York, Routledge, 1993. Longino, $H_{\text {., "Cognitive and }}$ Non-cognitive Values in Science:Rethinking the Dichotomy", en Nelson, L. H. and Nelson, J. (eds) Feminism, Science, and Philosophy of Science. ,G. Bretain, Kulver Academic Publishers, 1996, pp. 39-58.

6 Longino, H., (1990), p. 216.

7 Véase Longino, H., (1990), pp. 5-6, donde señala: « Mi principal objetivo es mostrar cómo los valores sociales juegan un rol en la investigación científica analizando aspectos del razonamiento cientificon. Longino, H., (1990), p. 3. Para la cuestión de la buena y la mala ciencia véase, sobre todo, el apartado del mismo libro "Debates About Science and Social Values», pp. 7-12.

8 Longino, H., (1990), pp. 12-13

9 Longino, $H_{.,}(1990)$, p. 216.

10 Longino, H., (1990) cap 3, pp. 38-61. igualmente en el cap. 10. Longino manifiesta: "Yo arguyo que el razonamiento evidencial depende siempre del contexto, que los datos constituyen evidencia a favor de una hipótesis solo a la luz de los supuestos de trasfondo que afirman una conexión entre el tipo de cosas o eventos de los que los datos son datos y el proceso o estado de hechos descrito por la hipótesis». Longino, H., (1990), pp. 215216.
11 Longino mantiene: "Asi un estado de cosas dado ...puede ser tomado como evidencia a favor de hipótesis muy diferentes e incluso conflictivas dadas determinadas creencias de base, apropiadamente conflictivası. Longino, H., (1990), pp. 43. Y más adelante: «El apoyo evidencial de una hipótesis es una cuestión relativa", depende del contexto en que se dan las creencias y supuestos. Longino, H., (1990), p. 45. Analiza varios ejemplos de la historia de la ciencia desde este punto de vista. Véase también, opus cit, pp. 41-42. p. 2

12 Longino, $H_{.,}(1990)$, p. 4.

13 Longino, $H_{.},(1990)$, p. 223. También p. 83.

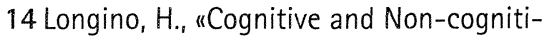
ve Values in Science:Rethinking the Dichotomy", en Nelson, L. H. and Nelson, J.(eds) Feminism, Science, and Philosophy of Science. ,G. Bretain, Kulver Academic Publishers, 1996, pp. 39-58.

15 Véase Laqueur, T., La construcción del sexo: cuerpo y género desde los orígenes hasta Freud. Madrid, Ed. Cátedra, 1994, p. 256.

16 Según Darwin, en el caso de la especie humana, los machos desarrollaron no solo tamaño, fuerza, coraje y aspecto físico sino también, razón, invención o imaginación. Era este un maravilloso resultado del éxito masculino en la lucha sexual en términos de la cual, el hombre se hace finalmente superior a la mujer. Darwin afirma que "de todas maneras es probable que e hombre sea tan superior en dotación mental a la mujer como el pavo real en plumaje ornamental a la pava realı. Citado en Romanes, G. L., "Mental Differences between Men and Women". Nineteenth Century, 21, (1887), p. 661. El origen del hombre, (2 vol). Madrid, M. E. Editores, 1994; ver sobre todo los capitulos finales del vol 2, pp. 527-574, en los que Darwin considera explicitamente a los hombres mejor dotados de "las facultades mentales superiores" observación, raciocinio e imaginación.

17 Spencer, H., "Psychology of the Sexes", Popular Science Monthly 4, (1873), pp. 3132.

18 Ferneseed, F., "Sexual Distinctions and Resemblances", Quaterly Journal of Sciences, 3, (1881) 
19 Ferrero, G., "The Problem of Woman from a Bio-Sociological Point of Viewn, The Monist, 4, (1893-94).

20 Mill, S. The Subjection of Woman , London, Melburne and Toronto, Ed. Everyman's Library, 1977, pp. 219-266.

21 Véase, Miller, Jacoby, R., "La ciencia y el papel de los sexos en la era victorianan, en López-Fajul de Argüelles (ed), La biología como arma social. Madrid, Ed. Alhambra, 1982. Maynard, Smith, J., Acerca de la evolución. Madrid, Blume Ediciones, 1979.

22 Spencer establece analogias entre salvajes, niños y mujeres aceptando la tesis de Ernest Haeckel ( biólogo alemán) propuesta en 1866, de que la ontogénesis recapitula la filogénesis (el embrión pasa por las diferentes etapas de la evolución de las especies y la filogénesis juega algún papel causal directo en su desarrollo ontológico). La teoría de la recapitulación es atractiva y tuvo gran éxito en su momento. Tuvo aplicaciones racistas y sexistas. La idea central es que los embriones hembras eran menos diferenciados, igual que los de los primitivos. La naturaleza de la mujer era menos especifica, más genérica y uniforme. La ontogénesis mostraba que ambos estaban más cerca de los antepasados de la especie (primates). En la filogénesis de las razas humanas, mujeres y salvajes ocupaban el rol del niño. Ambos eran eslabones inferiores en la gran cadena de la evolución. Eran inmaduros e imperfectos, etapas previas al desarrollo que alcanza el hombre blanco adulto. Un interesante debate sobre la actualidad de este tema se ha desarrollado en Hopos-Line fundamentalmente el 23y 24 de agosto de este año.

23 Spencer, H., "Psychology of the Sexes", Popular Science Monthly 4, (1873), pp. 3132.

$24 \mathrm{lbidem}$. Las cursivas son mias.

$25 \mathrm{lbidem}$

26 lbidem.

27 Véase Russett, C. E. Sexual Science: The Victorian Construction of Womanhood Cambridge, Harvard Univ. Press. 1991, pp. 110-117.

28 Ibidem.
29 La medicina corrobora esta creencia, pues los médicos, (y destacadamente en Norteamérica donde la mujer estaba teniendo acceso a la educación superior y el trabajo) mantienen virulentamente que la naturaleza femenina, su fisiologia y anatomía se resentia radicalmente si ejercia una función que no fuese la reproductora. Por tanto, la educación y el trabajo atentan contra su naturaleza, la disminuye y enferma; ni sus sistema nervioso, ni su cerebro, ni su organismo en general, está preparado para el esfuerzo que el trabajo fuera de casa o el estudio serio y continuado exigen.

30 De Beauvoir, S., El segundo sexo, Aguilar (Obras completas), 1981. p. 48.

31 Spencer, Opus cit. p. 32

32 La frenología se constituye a partír de los trabajos de John Franz Gall (1758-1828). Gall era un anatomista fisiologista y antropólogo alemán que colaboró Von Hermman Helmholtz (1776-1823) un gran experimentador en fisiología. De él tomo la idea de que sólo podian existir en la naturaleza procesos causales de carácter fisico y que la fisiología era la única ruta para establecer el estudio científico de la naturaleza humana. Entiende la nueva fisiología como el medio para construir el estudio cientifico de las diferencias humanas, tal como expone en su obra Anatomie et physiologie du systême nerveux... (4vol). Esta obra que realizó conjuntamente con J. C. Spurzheim, fue editada en Paris en Schoell entre 1810-1819. Otras obras suyas son Sur les fonctions du cerveau et sur celes de chacune de ses parties, (6 vol), Paris, Baillére, 1822-1825; On the fuctions of brain and of each of its parts, (6 vol), Boston, Marsh, Capen and Lyon, 1835.

33 Sus prácticas fueron mal vistas en Viena y tres años después fue forzado a dejar el pais.

34 Gall creia que la mente humana constaba de 37 facultades diferentes (desde las emotivas a las de identidad e intelectivas).

35 J. C. Spurzheim(1776-1832) escribio The Physiognomical System of Drs Gall and Spurzheim, (2 v). London, Baldwin, Gradock and Joy, 1815 y The Anatomy of the Brain, with a General View of Nervous System, London, Highley, 1826.
36 Paul Broca, (1824-1880), neurólogo y gran antropólogo francés. Fue fundador de Annals of Antropological Review y de dibersos artículos y libros, como, por ejemplo, Remarks on the seat of faculty of articulate language, followed by an observation of aphemia, reeditado en $\mathrm{G}$. von Bonnis, Same Papers on the Cerebral Cortex, Springfield, Thomas, 1960, pp. 49-72. Sobre Broca véase, Schiller, F., Paul Broca: Founder of French Anthropoly, Explorer of the Brain. Oxford, University Press, 1992. También Sagan, C., El cerebro de Broca: reflexiones sobre el apasionante mundo de la ciencia. Barcelona, Crítica, 1999.

37 Broca, P., Antropological Review 6, (1868).

38 Estas mediciones se aplican también a los idiotas, imbéciles, criminales, enfermos. Hay interés en establecer su tipología.

39 MacGrigor, J., "On the real Differences in the Minds of Men and Women", Journal of Anthropological Society of London, 7 (1869).

40 Concepción Arenal argumenta de manera admirablemente lúcida en contra de los resultados señalados por los estudios de cerebro centrando su critica en las tesis de Gall. Ella afirma, por ejemplo, que, si se supone que el sistema nervioso femenino es más irritable entonces tiene más actividad, por tanto se pregunta ¿no podria su cerebro hacer el mismo trabajo intelectual con menos volumen? ¿No vemos hombres más inteligentes que otros cuya frente es menor? Cualquiera que haya observado cabezas y comparado inteligencias, ¿puede dudar de que en muchos casos la calidad de la masa cerebral suple la cantidad? Además lo que cuenta es el volumen relativo no el absoluto. Lo que se observa es que en la mayor parte de las facultades la mujer es igual al hombre; la diferencia intelectual sólo empieza donde empieza la educación. Lo que necesita la mujer como el hombre, es ejercitar sus facultades a través de la educación. Nada autoriza a afirmar que la inferioridad intelectual de la mujer sea orgánica. Arenal, C. La emancipación de la mujer en España. Madrid, Ed. Jucar, 1974, pp. 106-111.

41 Moebius, P. J., La inferioridad mental de la mujer. Barcelona, Bruguera, 1982. 
42 Hay que señalar que la teoria de Moebius acerca de la inferioridad mental de las mujeres se hace extensiva a la cuestión racial ya que las mismas diferencias en las circunvoluciones cerebrales encontradas entre los sexos se dan entre las razas. Moebius afirma, que " en los hombres poco desarrollados en la parte mental (un negro por ejemplo), [Rüdinger] encuentra los mismos datos anatómicos hallados en el lóbulo parietal de la mujer...n Moebius, P. J. (1982), p. 7. Además Moebius habia realizado investigaciones en las que trataba de mostrar la correlación entre ciertas peculiaridades morfológicas del cerebro y la excelencia de ciertas facultades (como estaban haciendo los evolucionistas etc.). Moebius "quería demostrar que el talento matemático se manifiesta en una preponderancia de la corteza supraorbital». Klara Müller, "Junge Schweiz, n 3". en. Moebius, P. J. (1982), p. 129.

43 Moebius, P. J., (1982) todas las citas de p. 7.

44 Moebius, P. J. (1982), p. 8

45 Moebius, P. J. (1982), p. 7

46 Moebius,P. J. (1981), p. 5.

$47 \mathrm{lbidem}$.

48 Moebius, P. J., (1982),p. 6. Se refiere al trabajo de Lombroso y Ferrero, La mujer como delincuente y prostituta.

49 Moebius, P. J.,(1982), p. 6.

$50 \mathrm{lbidem}$.

51 Moebius,P. J. (1982), p. 8.

52 Moebius, P. J., (1982), p. 8. Platón en la República (V) ya sostenía esta idea al mantener que los hombres siempre harán mejor cualquier cosa de las que hagan las mujeres, excepto las tareas domésticas. Emprendan lo que emprendan lo harán menos bien. Sócrates pregunta a Glaucón: ¿conoces alguna profesión humana en la que el género masculino no sea superior al género femenino en todos los respectos? A lo que responde "es verdad que prácticamente en todas las cosas uno de los sexos es muy superior al otron. Platón, El Político, 455 c-d.

53 Moebius,P. J. (1982), p. 9.

54 lbídem.

55 lbidem.

56 Ibidem.

57 lbidem

58 Moebius,P.J. (1982), p. 10.

$59 \mathrm{lbidem}$.

60 Moebius,P.J. (1982), p. 9.

61 Moebius,P. J., (1982), p. 11
62 Ibídem.

63 Ibídem.

64 Moebius,P. J.,(1982), p. 13.

65 Moebius, P. J., (1982),p. 14.

66 Moebius,P. J., (1982), p. 15.

67 Moebius,P. J., (1982), p. 14.

68 lbídem.

69 Moebius,P. J. (1982), p. 12.

70 lbídem.

71 Moebius,P. J., (1982), p. 15.

72 lbídem.

73 Moebius,P. J., (1982), p. 16.

74 Moebius,P. J., (1982), p. 17.

75 lbídem.

76 Moebius, P. J., (1982), p. 18.

77 Moebius, P. J., (1982),p. 13.

78 Moebius,P. J., (1982), p. 16.

79 lbídem.

80 Moebius, P. J. (1982), p. 17.

81 lbídem.

82 Moebius,P. J., (1982), p. 18.

83 Como muy bien señala la traductora de la obra de Moebius, Franca Ongaro Basaglia, en la introducción (pp. XII-XIII).

84 Por ejemplo, Klara Müller afirma: "En cuanto a las verdades anatómicas de Moebius, sólo cabe señalar que pertenecen a una época preanatómica que no están comprobadas y que no pueden ser trasladadas sin más ni más al campo de la psicologia». Müller, K., "Junge Schweiz, No 3" en Moebius, P. J., (1982), p. 130.

85 Müller, K., Junge Schweiz, n 3. 1900, Das magazin für Literatur. Berlin, 5. I. 1901. Dohm, H., Die Frau. Berlin junio 19001. The Englisshwoman's Review. Die Frauenbewegung. II. 3. 4.5.1901. Todos ellos como apéndices del libro de Moebius, pp. 129-154.

86 Muller, K., Junge Schweiz, no 3. 1900 en Moebius, P.J., (1982) p. 130. Y continúan afirmando que el mismo Moebius, en otro momento de su trabajo científico, "tampoco hubiera afirmado saber en qué consiste la diferencia entre el cerebro masculino y el femenino.

87 Véase apéndices en Moebius, P. J., (1982), pp. 33-116.
88 Las críticas favorables son de Wulff Oldenburg, Doctor L., K., Hartmann. Aparecidos en Reichs-Medizinal-Anzeiger ${ }_{i}$ XXVI. $n^{\circ} 2$ del 18.1.01. Frankfurter Schulzeitung, Frankfurt a. M. 1 de noviembre de 1902. Nord un Süd febrero de 1901. Cuaderno 287. Kreuz-Zeitung, Berlin, 25 de junio de 1901. Der litterat. Revista para literatura y arte moderno. Braunschweig. Diario de H. Hansjakob, Stuttgart 1902, p. 346.

89 Dorinda Outran, The Body and the French Revolution, New Haven, Yale Univer. Press, p. 156.

90 Tresguerres, J. A. F., „Procesos de diferenciación sexual», en Botella Llusiá J.y Tresguerres, J. A: F. (directores), Hormonas, instintos y emociones. Madrid, Editorial Complutense, 1996, pp. 11-12 las cursivas son mías).

91 Véase, por ejemplo, Susana Medina Salem, "Ciencia e ideologia: sesgos heteropatriarcales en la construcción de la identidadı. Laguna. Revista de Filosofía. no VII, 2001, en prensa.

ARBOR CLXXXI 716 NOVIEMBRE-DICIEMBRE (2005) 479-492 ISSN: 0210-1963 